\title{
Comparação de metodologias de preenchimento de falhas de séries históricas de precipitação pluvial anual
}

\author{
Luiz F. C. de Oliveira ${ }^{1}$, Ana P. Fioreze ${ }^{2}$, Antonio M. M. Medeiros ${ }^{3} \&$ Mellissa A. S. Silva ${ }^{4}$
}

\begin{abstract}
RESUMO
A falta de registro de dados de precipitação em estações pluviométricas relativa a problemas com os aparelhos de coleta ea ausência do operador em determinadas épocas, comprometem a continuidade das informações. Para que se possa aplicar um tratamento está́stico em uma série histórica de precipitação devese proceder ao preenchimento das fal has existentes. Os métodos mais usuais nesta etapa são: ponderação regional (PR), regressões linear (RM) e potencial (RP) múltiplas, ponderação regional com base em regressões lineares (RS) e vetor regional (VR). Empregando-se séries históricas de precipitação total anual com 22 anos de observação para 6 estações pluviomé tricas localizadas no Estado de Goiás, fez-se uma análise comparativa entre os métodos de preenchimento de falha e se avaliaram os desvios relativos entre os valores observados e os estimados pelos métodos empregados. No método do vetor regional foram consideradas, ainda 5 possibilidades, ou seja, extraindo-se da série o dado observado substituindo-se, na série, o valor estimado pelos métodos da ponderação regional (VR/PR), regressão linear (NR/RM), regressão potencial (VR/RP) e ponderação regional com base em regressões lineares (VR/RS). Pelos resultados obtidos pode-se concluir, que em ordem crescente, que os menores desvios relativos foram obtidos pelos métodos: RM, VR/RP, PR, VR/RM, VR/RS, RP, RS, VR/PR e VR. Com relação à metodologia do vetor regional, obtevese uma melhoria nas estimativas do preenchimento das fal has quando se empregou a combinação com os demais métodos.
\end{abstract}

Palavras-chave: vetor regional, ponderação regional, regressão múltipla

\section{Comparison of gap filling methodologies of annual historical series of rainfall}

\begin{abstract}
The absence of rainfall data for stations due to equipament or operator problems compromise information continuity. In order to apply a statistical treatment on rainfall historical series, gap filling needs to be done. Usual methods for this are: regional means (PR), linear (RM) and potential (RP) multiple regression, regional means using linear regression (RS) and regional vector (VR). Using total annual rainfall historical series with 22 years of observation for six rainfall stations in the State of G oias, a comparison analysis was done between gap filling methods and the differences between observed and estimated values were evaluated. For the regional vector method five possibilities were considered extracting from the series the observed data, replacing the estimated value in the series by the methods of regional means (VR/PR), linear regression (VR/RM), potential regression (VR/RP) and regional means using linear regression (VR/RS). According to results it was concluded, in ascending order, minor the lowest differences in relative desviation were obtained with the methods: $R M, V R / R P, P R, V R / R M, V R / R S, R P, R S, V R / P R$ and $V R$. According to the regional vector method, an improvement was verified in data values was verified when a combination between some other methods was used.
\end{abstract}

Key words: regional vector, regional means, multiple regression

\footnotetext{
${ }^{1}$ UFLA/UFG, Bolsista em Produtividade do CNPq, Departamento de Engenharia/UFLA, Campus Universitário, CP 3037, CEP 37200-000, Lavras, MG. Fone: (35)3829-1679. Email: coutinho@ deg.ufla.br

2 UFG, Campus Samambaia, CP131, CEP 74001-970, Goiânia, GO. Fone: (62) 3521-1540, Email: anafioreze @ semarh.goias.gov.br

${ }^{3}$ Doutorando em Solo e Água UFG, Bolsista CNPq, Campus Samambaia, CP131, CEP 74001-970, Goiânia, GO. Fone: (62) 3521-1540, Email: amarcosmedeiros@yahoo.com.br

${ }^{4}$ UFG, Campus Samambaia, CP131, CEP: 74001-970, Goiânia, GO. Fone: (62) 3521-1540, Email: melsoler@gmail.com
} 


\section{INTRODUÇÃO}

O conhecimento acerca do regime hídrico em uma bacia hidrográfica é fundamental nos estudos hidrológicos que servem como base para projetos de diferentes usos de água, tornando-se fator indispensável para um gerenciamento adequado dos recursos hídricos (Santos et al., 2009). A Lei nº9433, de 1997, que institui no Brasil a política nacional de recursos hídricos preconiza, dentre os instrumentos de gestão, o sistema de informações hidrológicas, porém este instrumento de gestão ainda está muito incipiente com relação aos demais existentes (Fioreze \& Oliveira, 2010).

A Agência Nacional das Águas disponibiliza as séries pluviométricas pelo sistema de informações hidrológicas Hidro Web, nas quais se observam falhas diárias, mensais e anuais, muitas vezes inviabilizando a utilização dessas séries. A existência de falhas nas séries históricas se deve, basicamente, à ausência do observador, falhas nos mecanismos de registro, perda das anotações ou das transcrições dos registros pelos operadores e encerramento das observações entretanto, como existe a necessidade de se trabalhar com séries contínuas, essas falhas necessitam ser preenchidas (Streck et al., 2009; Bertoni \& Tucci, 2007). Para o preenchimento das falhas nas séries históricas, Leivas et al. (2005) recomendam a seleção das estações meteorológicas da mesma região ecoclimática e altitude semelhante, caracterizando-a como hidrologicamente homogênea.

Uma forma comum de se obter dados sintéticos para um local específico com pequenas séries de dados observados a fim de se estender o período de observação ou corrigir falhas, é a utilização de modelos hidrológicos (Alexandre, 2008). A dificuldade para a utilização de modelos hidrológicos decorre das limitações referentes aos dados disponíveis, tanto em termos de densidade da rede de coleta de dados como em relação ao pequeno período de observações e das falhas existentes nas séries (Silva et al., 2002; Oliveira et al., 2008a; 2008b).

Em hidrologia, as séries históricas de precipitação são informações fundamentais para o entendimento do regime hidrológico (Mello \& Silva, 2005). O emprego de dados obtidos a partir de geradores de precipitação em modelos de simulação hidrológica, principalmente naqueles utilizados na predição das taxas de escoamento superficial e de perdas de solo, tem-se intensificado nos últimos anos. Esses geradores, desenvolvidos, em geral, a partir de séries históricas disponíveis, são avaliados em função da capacidade que apresentam para gerar dados com estatísticas similares às observadas nos dados históricos (Oliveira et al., 2005).

Considera-se, desta forma, que a aplicação da modelagem no preenchimento de falhas pode ser uma ferramenta útil nos estudos de gestão de recursos hídricos, facilitando o conhecimento do comportamento hidrológico permitindo, assim, um aproveitamento consciente dos recursos hídricos (Barnetche \& Kobiyama, 2006).

A regionalização hidrológica é, nesses casos, uma das formas encontradas para transferir informações de locais com registros existentes para locais onde não existem dados. Procura-se, nos modelos de regionalização de vazão, correlacionar o escoamento superficial gerado em uma bacia com vari- áveis explicativas, sobretudo as climáticas e morfométricas da bacia hidrográfica. Segundo Tucci (2002), a precipitação pluvial é uma das principais variáveis explicativas empregadas nos modelos de regionalização de vazão, sendo a precipitação média anual a mais utilizada.

Para iniciar o estudo hidrológico de uma região faz-se uma análise das séries históricas das estações climatológicas, pluviométricas e fluviométricas (Chibana et al., 2005). Conforme Bertoni \& Tucci (2007) e Lemos Filho et al. (2007), as análises preliminares a serem efetuadas nas séries históricas consistem no preenchimento de falhas e na verificação da consistência, ou seja, do grau de homogeneidade dos dados disponíveis em uma estação de coleta com relação às observações registradas em estações vizinhas.

Segundo Zeilhofer et al. (2003) para o preenchimento de falhas nas séries temporais das vazões diárias e da precipitação deve-se escolher, em cada execução, pares de pontos com correlação máxima, definindo uma regressão linear entre os conjuntos de dados permitindo, assim, preencher a falha empregando-se o modelo ajustado.

De acordo com Chibana et al. (2005) vários métodos podem ser utilizados no preenchimento de falhas de dados meteorológicos, incluindo-se a utilização de médias de dados observados ou dados sintéticos obtidos de geradores de dados. Fill (1987) e Bertoni \& Tucci (2007) apresentam várias metodologias empregadas no preenchimento de falhas e comentam que nenhuma se presta ao preenchimento de falhas diárias, sendo mais recomendadas no preenchimento de falhas mensais ou anuais.

Com o objetivo de comparar as metodologias de preenchimento de falhas disponíveis na literatura, empregaram-se séries históricas de precipitações totais anuais com 22 anos contínuos de observações em 6 postos de coleta de dados, localizadas na região central do Estado de Goiás.

\section{MATERIAL E MÉTODOS}

Para se proceder à análise comparativa das metodologias de preenchimento de falhas, selecionaram-se 6 estações pluviométricas da rede da Agência Nacional de Águas, localizadas na região central do Estado de Goiás. As estações estão inseridas na bacia do rio Paraná, na sub-bacia do rio Meia Ponte, tributário do Rio Paranaíba. Na Tabela 1 se encontram as estações selecionadas, o número de identificação das estações, os municípios, as coordenadas geográficas e os anos de registro extraídos do Hidro Web. As distâncias radiais entre as estações medidas sobre a superfície da Terra, variam entre 18,7 e 63,1 km, entre as estações Emgopa e Goiânia, e Aragoiânia e Inhumas, respectivamente. Como os anos de observação das estações selecionadas são variáveis, foram selecionados os anos comuns de registros pluviométricos perfazendo, assim, as séries de totais anuais com 22 observações.

Com o intuito de se verificar a homogeneidade das séries anuais de precipitação pluvial, fez-se a análise de consistência para cada estação isoladamente, empregando-se a metodologia da dupla massa descrita por Bertoni \& Tucci (2007). 
Tabela 1. Estações pluviométricas da rede da Agência Nacional de Águas empregadas no estudo

\begin{tabular}{|c|c|c|c|c|c|c|}
\hline Estação & Identificação ANA & Nome & Município & Latitude & Longitude & Anos das séries \\
\hline P1 & 1649002 & Emgopa & Goiânia & $16042^{\prime} 4^{\prime \prime}$ & $4905^{\prime} 42^{\prime \prime}$ & 1969 a 1997 \\
\hline P2 & 1649001 & Aragoiânia & Aragoiânia & $16054^{\prime} 43^{\prime \prime}$ & "4902 $27^{\prime \prime}$ & 1974 а 2007 \\
\hline P3 & 1649005 & Goiânia & Goiânia & $16040^{\prime} 1^{\prime \prime}$ & 49o 16' 1" & 1974 a 2002 \\
\hline P4 & 1649006 & Inhumas & Inhumas & $16020^{\prime} 45^{\prime \prime}$ & $49029^{\prime} 38^{\prime \prime}$ & 1969 a 2006 \\
\hline P5 & 1649012 & Trindade & Trindade & $16039^{\prime} 32^{\prime \prime}$ & $49029^{\prime} 10^{\prime \prime}$ & 1974 a 2007 \\
\hline P6 & 1649004 & Goianápolis & Goianápolis & $16030^{\prime} 58^{\prime \prime}$ & 490 1' 12" & $\begin{array}{c}1974 \text { a 1989, } 1991 \text { e } \\
1993 \text { a } 2006\end{array}$ \\
\hline
\end{tabular}

Neste método se selecionam a estação de interesse e as mais próximas, cujos totais anuais acumulados se pretende avaliar a consistência dos dados, são plotados em um gráfico nas ordenadas e nas abscissas os totais médios anuais das demais estações. Haverá consistência dos totais anuais da estação analisada quando houver uma tendência linear em relação às estações vizinhas. A verificação da linearidade entre os totais anuais da estação analisada com relação às demais é avaliada pelo ajuste da equação da reta e do coeficiente de determinação, obtidos pela técnica da minimização da soma dos quadrados dos desvios.

Visando à análise do preenchimento de falhas nas séries dos totais anuais das 6 estações pluviométricas, procedeuse da seguinte forma: retirou-se, para cada estação e ano de observação, o valor conhecido; preencheu-se a falha empregando-se as diferentes metodologias e se verificaram os desvios relativos entre o valor observado e os estimados pelas diferentes metodologias. Realizaram-se, enfim, para cada metodologia empregada, 132 simulações de preenchimento de falhas.

Os métodos empregados no preenchimento de falhas foram os da: ponderação regional, regressão linear, ponderação regional com base em regressões lineares e vetor regional. No método da ponderação regional, descrito por Bertoni \& Tucci (2007) e Alves et al. (2006), a falha da precipitação anual na estação de interesse é estimada pelo emprego da seguinte equação:

$$
\mathrm{P}_{\mathrm{x}}=\frac{1}{\mathrm{n}} \sum_{\mathrm{i}=1}^{\mathrm{n}} \frac{\mathrm{N}_{\mathrm{x}}}{\mathrm{N}_{\mathrm{i}}} \mathrm{P}_{\mathrm{i}}
$$

em que:

$\mathrm{P}_{\mathrm{x}}=$ precipitação anual da estação a ter a falha na série preenchida $(\mathrm{mm})$

$P_{i}=$ precipitação anual da estação vizinha de ordem " $i$ " para o ano em que se verificou a falha $(\mathrm{mm})$

$\mathrm{N}_{\mathrm{x}}$ e $\mathrm{N}_{\mathrm{i}}=$ precipitações médias anuais das estações a ter a falha na série preenchida e da vizinha de ordem "i", respectivamente $(\mathrm{mm})$

$\mathrm{n}=$ número de estações vizinhas

Segundo Tucci (2002) e Bertoni \& Tucci (2007), o método de preenchimento de falha pela regressão linear é mais aprimorado em comparação com a ponderação regional. Neste método se emprega a regressão linear múltipla correlacionando-se a estação com falha com as estações vizinhas, ou seja:

$$
\mathrm{P}_{\mathrm{x}}=\mathrm{a}_{0}+\sum_{\mathrm{i}=1}^{\mathrm{n}} \mathrm{a}_{\mathrm{i}} \mathrm{P}_{\mathrm{i}}
$$

em que:

$a_{o}$ e $a_{i}=$ coeficientes de ajuste do modelo linear

Outra metodologia alternativa usada no preenchimento de falhas de série apresentada por Bertoni \& Tucci (2007) é o modelo do tipo potencial, dado por:

$$
\mathrm{P}_{\mathrm{x}}=\mathrm{a}_{0} \prod_{\mathrm{i}=1}^{\mathrm{n}} \mathrm{P}_{\mathrm{i}}^{\mathrm{a}_{1}}
$$

Ajustaram-se 23 modelos lineares e potenciais múltiplos, considerando-se todos os anos de observação das séries dos totais anuais e 22 ajustes, retirando-se um ano da série; posteriormente, foram calculados os valores médios dos coeficientes do modelo linear; para se efetuar o ajuste dos 23 modelos lineares e potenciais múltiplos, empregou-se a rotina Solver, da planilha Excel.

O método da ponderação regional com base em regressões lineares, segundo Pruski et al. (2004) e Bertoni \& Tucci (2007), é uma combinação dos métodos anteriores descritos. Este método consiste em estabelecer regressões lineares entre os postos com dados a serem preenchidos, $\mathrm{P}_{\mathrm{x}}$, e cada um dos postos vizinhos, $\mathrm{P}_{1}, \mathrm{P}_{2}, \ldots, \mathrm{P}_{\mathrm{N}}$. De cada uma das regressões lineares efetuadas obtém-se o coeficiente de correlação, sendo o preenchimento realizado com base na seguinte expressão:

$$
P_{x}=\frac{\sum_{i=1}^{n} r_{P_{x} P_{i}} P_{i}}{\sum_{i=1}^{n} r_{P_{x} P_{i}}}
$$

em que:

$r_{P_{x} P_{i}}$ - coeficiente de correlação linear entre os postos vizinhos

Ajustaram-se 15 equações lineares médias que correlacionam, em pares, as 6 estações pluviométricas empregadas neste estudo. Cada equação proveio da média do ajuste de 23 equações, considerando-se todos os anos de observação das séries dos totais anuais e 22 ajustes, retirando-se um ano da série.

Segundo recomendações de Barbosa et al. (2005) e Pruski et al. (2004), para a aplicação dos métodos da regressão linear e da ponderação regional com base em regressões lineares adotou-se, como critério mínimo, a obtenção de coeficiente de determinação superior a 0,7 .

$\mathrm{O}$ método do vetor regional segundo Keller Filho et al. (2005) e Bertoni \& Tucci (2007), consiste na determinação de uma série cronológica, sintética, de índices pluviométricos anuais, oriundos da extração por um método de máxima ve- 
rossimilhança da informação contida nos dados de um conjunto de estações agrupadas regionalmente. Esses índices são únicos para toda a região e estão relacionados às precipitações em cada estação por meio de coeficientes apropriados, de modo que as estimativas da precipitação anual, na estação "j” e no ano "i”, resultam do produto:

$$
\hat{\mathrm{P}}_{\mathrm{i}, \mathrm{j}}=\mathrm{L}_{\mathrm{i}} \mathrm{C}_{\mathrm{j}}
$$

em que:

$\mathrm{L}_{\mathrm{i}}$ - vetor coluna, com "n" linhas, também chamado vetor regional

$\mathrm{C}_{\mathrm{j}}$ - vetor linha, com "m" colunas

$\mathrm{n}$ - número de anos das séries de totais anuais

m - número de estações pluviométricas

$\mathrm{Na}$ estimativa do vetor regional empregou-se o método dos mínimos quadrados de forma a minimizar a soma dos quadrados dos desvios, ou seja:

$$
\mathrm{SQD}=\left(\mathrm{P}_{\mathrm{i}, \mathrm{j}}-\hat{\mathrm{P}}_{\mathrm{i}, \mathrm{j}}\right)^{2}
$$

Para tal se emprega um processo iterativo assumindo-se como estimativa inicial dos elementos do vetor regional, para cada ano, a média das precipitações das "m" estações que compõem a série, ou seja:

$$
\mathrm{L}_{\mathrm{i}}^{0}=\frac{1}{\mathrm{~m}} \sum_{\mathrm{j}=1}^{\mathrm{m}} \mathrm{P}_{\mathrm{i}, \mathrm{j}} \text { para } \mathrm{i}=1 \mathrm{a} \mathrm{n}
$$

Com a estimativa inicial se aplica para cada estação “j”, o método dos mínimos quadrados resultando:

$$
\begin{gathered}
C_{j}=\frac{\sum_{i=1}^{n} L_{i}^{0} P_{i, j}}{\sum_{i=1}^{n}\left(L_{i}^{0}\right)^{2}} \text { para } j=1 \text { a m } \\
L_{i}=\frac{\sum_{j=1}^{m} C_{j} P_{i, j}}{\sum_{i=1}^{m}\left(C_{j}\right)^{2}} \text { para } i=1 \text { a n }
\end{gathered}
$$

Utilizou-se, neste trabalho, um aplicativo desenvolvido em linguagem Delphi, por Medeiros \& Oliveira (2007) para se proceder à análise de consistência e ao preenchimento das falhas empregando-se o método do vetor regional.

Como proposta foram realizadas, também, simulações, empregando-se o método do vetor regional combinado com os métodos da ponderação regional, regressão linear, regressão potencial e da ponderação regional, com base em regressões lineares; para isto, em cada simulação se fez a substituição individual do valor a ser preenchido pelo obtido, pelos métodos da ponderação regional, regressão linear e da ponderação regional com base em regressões lineares perfazendo, portanto, 528 simulações.

\section{RESULTADOS E DISCUSSÃO}

A Figura 1 apresenta as curvas das duplas massas para todas as estações pluviométricas empregadas neste estudo, nas quais foi analisada, em cada estação, a consistência da série histórica com a média das estações vizinhas. Para todas as estações observa-se uma consistência das séries históricas com comportamento linear e os coeficientes de determinação próximos de 1o que, segundo Buriol et al. (2006) e Alves et al. (2006), garante a homogeneidade regional das estações selecionadas.

Os modelos lineares e potenciais múltiplos para a estação P1 (Emgopa) apresentaram, com relação às suas vizinhas, um bom ajuste com coeficiente de determinação acima de 0,7 conforme recomendação de Barbosa et al. (2005) e Pruski et al. (2004).

$$
\begin{aligned}
& \mathrm{P}_{1}=817,8685-1,1466 \mathrm{P}_{2}-0,3478 \mathrm{P}_{3}+0,1694 \mathrm{P}_{4}+0,87875 \mathrm{P}_{5}+ \\
& 0,92989 \mathrm{P}_{6} \quad \mathrm{r}^{2}=0,9325 \\
& \mathrm{P}_{1}=12,9262 \mathrm{P}_{2}^{-0,7399} \mathrm{P}_{3}^{-0,2646} \mathrm{P}_{4}^{0,2282} \mathrm{P}_{5}{ }^{0,5716} \mathrm{P}_{6}{ }^{0,8615} \\
& r^{2}=0,9460
\end{aligned}
$$

Os coeficientes dos modelos lineares e potenciais múltiplos provêm da média dos 23 ajustes considerando-se todos os anos da série e a retirada individual de cada ano da série. Os coeficientes de variação dos coeficientes ajustados ficaram na faixa de - $-0,28$ e 0,50 para o modelo linear e de $-0,38$ a 0,24 para o modelo potencial, indicando pequenas variações nos valores individuais dos coeficientes. Para ambos os modelos ajustados tem-se que algumas estações colaboram negativamente e outras de forma positiva, no preenchimento da falha, devido ao efeito regional da distribuição das chuvas junto às estações pluviométricas selecionadas.

A Tabela 2 apresenta as médias dos coeficientes lineares $\left(a_{o}\right)$ e angulares $\left(a_{1}\right)$, e dos coeficientes de correlação $(r)$, obtidos nos ajustes dos modelos lineares simples e os pares de estações pluviométricas. Os valores são médios de 23 ajustes ressaltando-se a série histórica completa e a retirada individual de cada ano da série, com coeficientes de variação na faixa de $-0,55$ a 0,62 . Observa-se bom ajuste dos modelos lineares com coeficientes de correlação acima de 0,84 , segundo a recomendação de Pruski et al. (2004).

De posse dos modelos lineares simples e múltiplos, procederam-se às análises de preenchimento de falhas extraindose, das séries históricas originais dos totais anuais, cada informação em separado. A Figura 2 apresenta, para cada estação pluviométrica, os desvios relativos médios entre os valores observados e os estimados pelas diferentes metodologias empregadas no preenchimento de falhas.

Analisando-se os desvios relativos médios entre os valores observados e os estimados pelas diferentes metodologias empregadas no preenchimento de falhas, pode-se constatar que, para as séries avaliadas em ordem crescente, os menores desvios relativos foram obtidos pelos seguintes métodos: regressão linear múltipla (RM), vetor regional combinado com a regressão potencial múltipla (VR/RP), ponderação regional (PR), vetor regional combinado com a regressão linear múltipla (VR/RM), vetor regional combinado com a regressão linear múltipla (VR/RS), regressão potencial múltipla (RP), ponderação regional com base em regressões lineares 
Emgopa

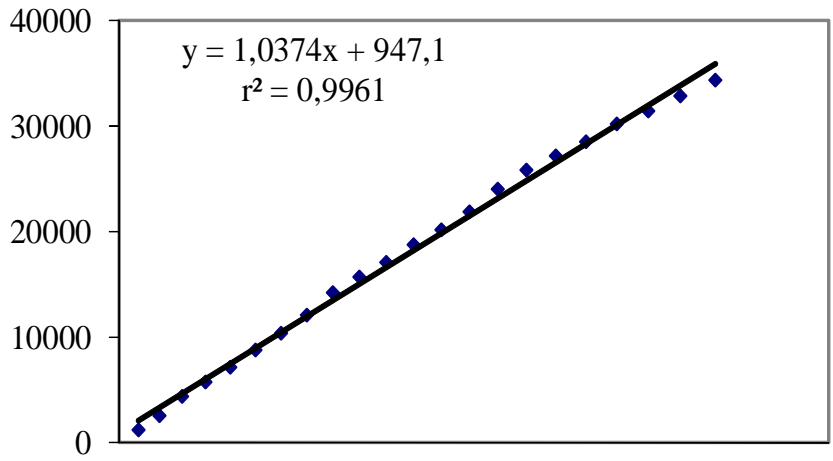

Goiania

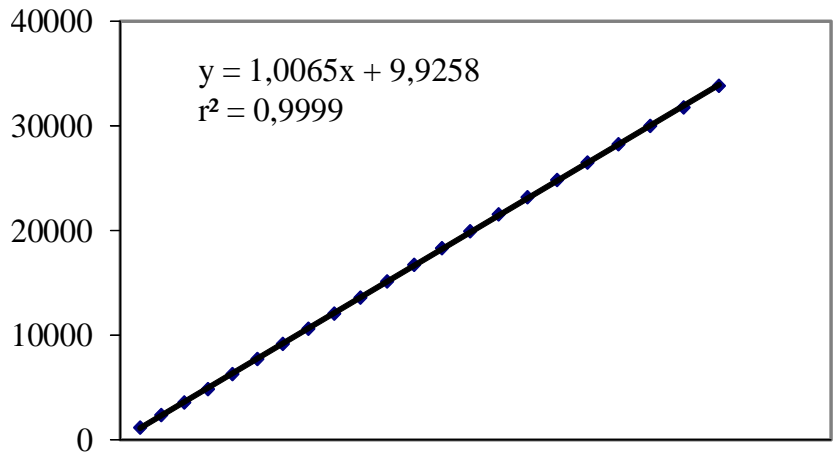

Trindade

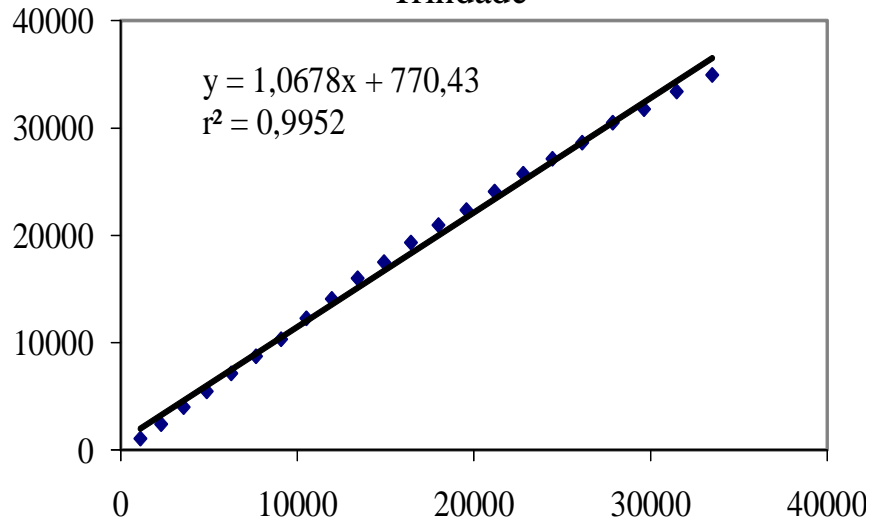

Aragoiania

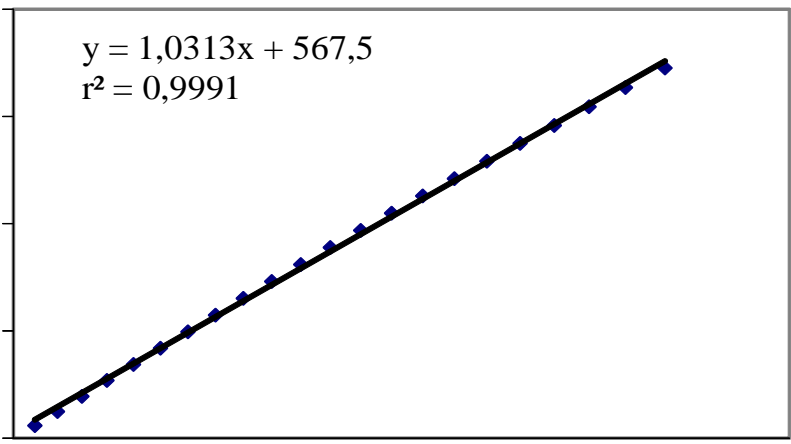

Inhumas

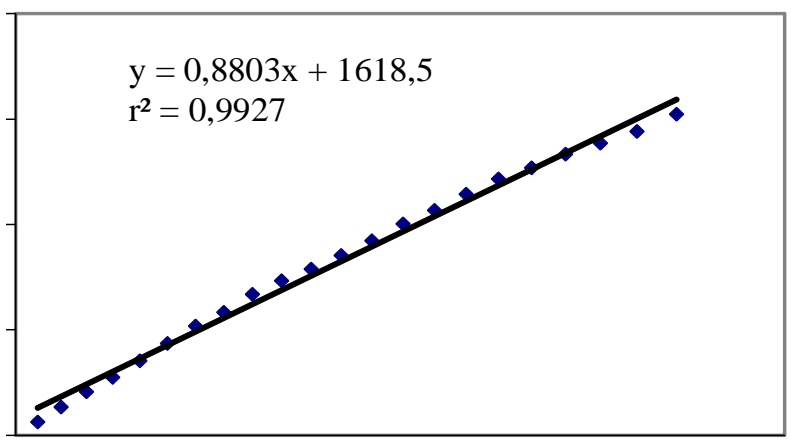

Goianápolis

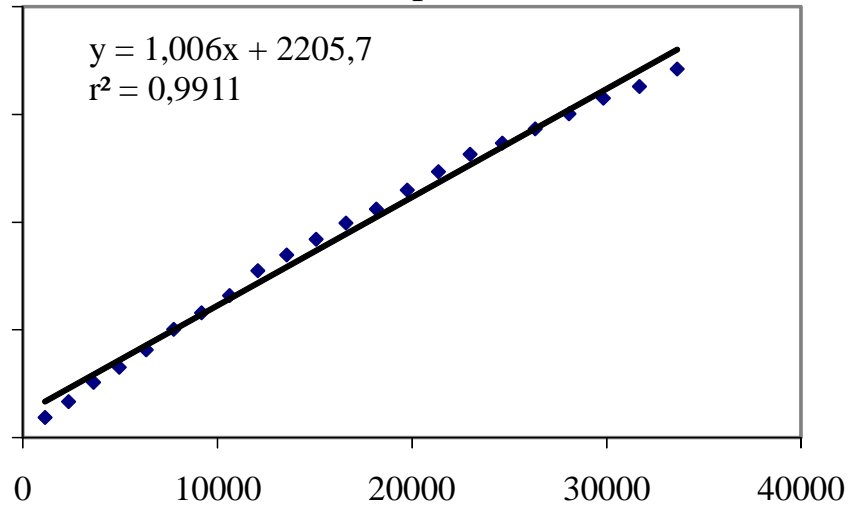

Figura 1. Curvas das duplas massas para verificação da consistência das séries históricas

(RS), vetor regional, combinado com a ponderação regional (VR/PR) e vetor regional (VR).

Pelos resultados obtidos para as estações avaliadas, consta-se que algumas metodologias mais simples (RM e PR) apresentaram melhor desempenho no preenchimento das falhas em relação aos métodos do vetor regional e ponderação regional com base em regressões lineares; não se pode afirmar, portanto, que essas metodologias possam ser empregadas sem prévia análise regional de seu desempenho.

Com relação à metodologia do vetor regional, obteve-se melhoria nas estimativas do preenchimento das falhas quando se empregou a combinação com os demais métodos. Os menores desvios relativos foram verificados quando se empregou a combinação dos métodos do vetor regional com a regressão potencial múltipla, com valor médio de $0,68 \%$. Os desvios relativos médios entre as combinações VR/RM e VR/ $\mathrm{RS}$, de 1,06 e 1,62\%, respectivamente, foram praticamente nulos, fato também constatado quando se compararam os desvios relativos obtidos entre as metodologias da ponderação regional com base em regressões lineares $(6,47 \%)$ e vetor regional combinado com a ponderação regional $(6,94 \%)$.

O método do vetor regional foi o que apresentou o maior desvio relativo médio $(10,35 \%)$ quando comparado com as demais metodologias; tal comportamento se deve ao fato de que, retirando-se cada valor da série histórica e simulando o valor a ser preenchido, dá-se uma alteração no valor médio para o ano em questão alterando, portanto, no vetor regional, assumido inicialmente no processo iterativo. A combinação do vetor regional com as outras metodologias tende a amenizar este problema. 
Tabela 2. Coeficientes lineares $\left(a_{0}\right)$, angulares $\left(a_{1}\right)$ e de correlação $(r)$, obtidos nos ajustes dos modelos lineares simples e os pares de estações pluviométricas

\begin{tabular}{|c|c|c|c|c|c|c|c|c|c|c|c|c|c|c|c|}
\hline & \multicolumn{3}{|c|}{$\mathbf{P}_{2}$} & \multicolumn{3}{|c|}{$\mathbf{P}_{3}$} & \multicolumn{3}{|c|}{$\mathbf{P}_{4}$} & \multicolumn{3}{|c|}{$\mathbf{P}_{5}$} & \multicolumn{3}{|c|}{$\mathbf{P}_{6}$} \\
\hline & $a_{0}$ & $a_{1}$ & $\mathbf{r}$ & $a_{0}$ & $a_{1}$ & $\mathbf{r}$ & $a_{0}$ & $a_{1}$ & $r$ & $a_{0}$ & $a_{1}$ & $\mathbf{r}$ & $a_{0}$ & $a_{1}$ & $\mathbf{r}$ \\
\hline$P_{1}$ & $-1611,15$ & 2,02 & 0,88 & $-152,23$ & 1,12 & 0,92 & $-78,27$ & 1,18 & 0,93 & $-181,57$ & 1,10 & 0,93 & 188,21 & 0,88 & 0,95 \\
\hline $\mathrm{P}_{2}$ & - & - & - & 601,66 & 0,66 & 0,92 & 663,58 & 0,65 & 0,92 & 543,30 & 0,65 & 0,98 & 805,91 & 0,49 & 0,94 \\
\hline $\mathrm{P}_{3}$ & - & - & - & - & - & - & 142,84 & 1,01 & 0,96 & 75,60 & 0,92 & 0,95 & 354,02 & 0,74 & 0,97 \\
\hline $\mathrm{P}_{4}^{3}$ & - & - & - & - & - & - & - & - & - & $-39,14$ & 0,90 & 0,97 & 316,75 & 0,69 & 0,94 \\
\hline $\mathrm{P}_{5}$ & - & - & - & - & - & - & - & - & - & - & - & - & $-375,01$ & 1,22 & 0,95 \\
\hline
\end{tabular}

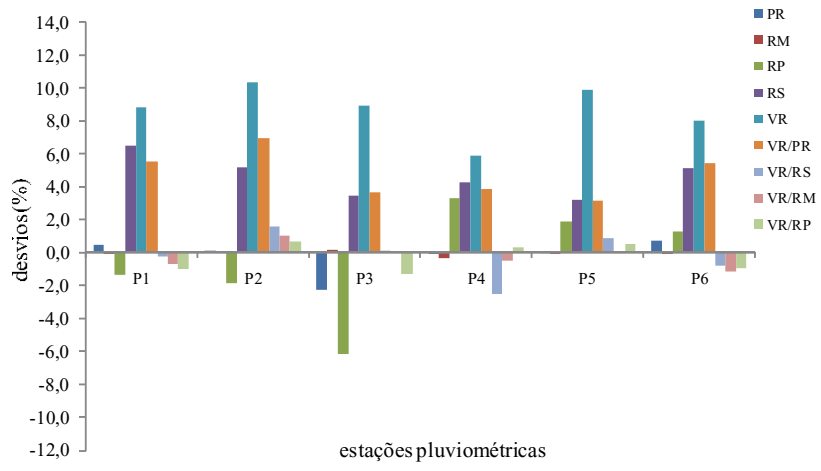

Figura2.D esvios relativos médios entre os valores observados e os estimados para as diferentes estações pluviométricas e metodologias empregadas no preenchimento de falhas

\section{CONCLUSÕES}

1. Os menores desvios relativos em ordem crescente foram obtidos pelos métodos da regressão linear múltipla, vetor regional combinado com a regressão potencial múltipla, ponderação regional, vetor regional combinado com a regressão linear múltipla, vetor regional combinado com a regressão linear múltipla, regressão potencial a, ponderação regional com base em regressões lineares, vetor regional combinado com a ponderação regional, vetor regional.

2. Ocorreu uma melhora nos resultados obtidos pelo método do vetor regional quando se fez a combinação desta metodologia com as demais.

\section{LITERATURA CITADA}

Alexandre, A. M. B. Modelagem hidrológica para o estado de Roraima. In: Simpósio de Recursos Hídricos do Sul, 17, 2008. São Paulo. Anais...Porto Alegre: Associação Brasileira de Recursos Hídricos, 2008. CD.Rom.

Alves, A.; Kobiyama, M.; Silva, R. V.; Checchia, T. Análise de dados hidrológicos na região do município de Alfredo Wagner/SC. In: Simpósio de Recursos Hídricos do Sul, 1, 2006, Curitiba. Anais...Porto Alegre: Associação Brasileira de Recursos Hídricos, 2006. CD-Rom.

Barbosa, S. E. da S.; Barbosa Júnior, A. R.; Silva, G. Queiroz da; Campos, E. N. B.; Rodrigues, V. de C. Geração de modelos de regionalização de vazões máximas, médias de longo período e mínimas de sete dias para a Bacia do Rio do Carmo, Minas Gerais. Engenharia Sanitária e Ambiental, v.10, n.1, p.64-71, 2005.
Barnetche, D.; Kobiyama, M. Aplicação do hycymodel no preenchimento de falhas de monitoramento de vazões. Geosul, v.21, n.42, p.185-194, 2006.

Bertoni J. C.; Tucci, C. E. M. Precipitação. In: Tucci, C. E. M. Hidrologia: Ciência e aplicação. Porto Alegre: UFRGS, 2007. p.177-241.

Buriol, G. A.; Estefanel, V.; Swarowsky, A.; D’Avila, R. F. Homogeneidade e estatísticas descritivas dos totais mensais e anuais de chuva de Santa Maria, Estado do Rio Grande do Sul. Revista Brasileira de Recursos Hídricos, v.11, p.89 97, 2006.

Chibana, E. Y.; Flumignan, D.; Mota, R. G.; Vieira, A. S.; Faria, R. T. Estimativa de falhas em dados meteorológicos. In: Congresso Brasileiro de Agroinformática, 9, 2005, Londrina. Anais...Londrina: SBI-AGRO, 2005. CD-Rom.

Fill, H. D. Informações hidrológicas. In: Barth, F. T.; Pompeu, C. T.; Fill, H. D.; Tucci, C. E. M.; Kelman, J. Braga Júnior, B. P. F. Modelos para gerenciamento de recursos hídricos. São Paulo: Nobel/ABRH, 1987. p.95-202.

Fioreze, A. P.; Oliveira, L. F. C. de. Usos dos recursos hídricos da bacia hidrográfica do ribeirão Santa Bárbara, Goiás. Pesquisa Agropecuária Tropical, v.40, n.1, p.30-38, 2010.

Keller Filho, T.; Assad, E. D.; Lima, P. R. S. de R. Regiões pluviometricamente homogêneas no Brasil. Pesquisa Agropecuária Brasileira, v.40, n.4, p.311-322, 2005.

Leivas, J. F.; Berlato, M. A.; Fontana, D. C. Risco de deficiência hídrica decendial na metade sul do Estado do Rio Grande do Sul. Revista Brasileira de Engenharia Agrícola e Ambiental, v.10, n.2, p.397-407, 2006.

Lemos Filho, L. C. de A; Carvalho, L. G. de; Evangelista, A. W. P.; Carvalho, L. M. T. de; Dantas, A. A. A. Análise espaço-temporal da evapotranspiração de referência para Minas Gerais. Ciência e Agrotecnologia, v.31, n.5, p.1462-1469, 2007.

Medeiros, A. M. M.; Oliveira, L. F. C. de. Programa para analise de consistência e preenchimento de falhas para dados de precipitação pluviométrica. In: Congresso Brasileiro de Engenharia Agrícola, 36, Bonito, 2007. Anais...Jaboticabal: SBEA, 2007, CD-Rom.

Mello, C. R. de; Silva, A. M. da. Métodos estimadores dos parâmetros da distribuição de Gumbel e sua influência em estudos hidrológicos de projeto. Irriga, v.10, n.4, p.318334, 2005.

Oliveira, L. F. C. de; Antonini, J. C. A.; Fioreze, A. P.; Silva, M. A. S. Métodos de estimativa de precipitação máxima para o estado de Goiás. Revista Brasileira de Engenharia Agrícola e Ambiental, v.12, n.6, p.620-625, 2008b.

Oliveira, L. F. C. de; Antonini, J. C. A.; Griebeler, N. P. Estimativas de chuvas intensas para o estado de Goiás. Engenharia Agrícola, v.28, n.1, p.22-33, 2008a. 
Oliveira, V. P. S.; Zanetti, S. S.; Pruski, F. F. Climabr parte I: Modelo para a geração de séries sintéticas de precipitação. Revista Brasileira de Engenharia Agrícola e Ambiental, v.9, n.3, p.348-355, 2005.

Pruski, F. F.; Pereira, S. B.; Novaes, L. F.; Silva, D. D.; Ramos, M. M. Precipitação média anual e vazão específica média de longa duração, na Bacia do São Francisco. Revista Brasileira de Engenharia Agrícola e Ambiental, v.8, n.2/3, p.247-253, 2004.

Santos, G. G.; Figueiredo, C. C. de; Oliveira, L. F. C. de; Griebeler, N. P. Intensidade-duração-frequência de chuvas para o Estado de Mato Grosso do Sul. Revista Brasileira de Engenharia Agrícola e Ambiental, v.13, p.899-905, 2009.
Silva, D. D.; Gomes Filho, R. R.; Pruski, F. F.; Pereira, S. B.; Novaes, L. F. Chuvas intensas no Estado da Bahia. Revista Brasileira de Engenharia Agrícola e Ambiental, v.6, n.2, p.362-367, 2002.

Streck, N. A.; Buriol, G. A.; Heldwein, A. B.; Gabriel, L. F.; Paula, G. M. de. Associação da variabilidade da precipitação pluvial em Santa Maria com a oscilação decadal do Pacífico. Pesquisa Agropecuária Brasileira, v.44, n.12, p.1553-1561, 2009.

Tucci, C. E. M. Regionalização de vazão. Porto Alegre: UFRGS, 2002, 256p.

Zeilhofer, P.; Lima, E. B. R.; Santos, F. M.; Rigo Júnior, L. O. Um ambiente SIG para modelagem integrada da qualidade da água utilizando Qual2e. Caminhos de Geografia, v.8, n.10, p.107-125, 2003. 